\title{
Interval-Valued Algebras and Fuzzy Logics
}

Bart Van Gasse, Chris Cornelis and Glad Deschrijver

\begin{abstract}
In this chapter, we present a propositional calculus for several intervalvalued fuzzy logics, i.e., logics having intervals as truth values. More precisely, the truth values are preferably subintervals of the unit interval. The idea behind it is that such an interval can model imprecise information. To compute the truth values of ' $p$ implies q' and ' $p$ and q', given the truth values of $p$ and $q$, we use operations from residuated lattices. This truth-functional approach is similar to the methods developed for the well-studied fuzzy logics. Although the interpretation of the intervals as truth values expressing some kind of imprecision is a bit problematic, the purely mathematical study of the properties of interval-valued fuzzy logics and their algebraic semantics can be done without any problem. This study is the focus of this chapter.
\end{abstract}

\section{Introduction}

Classical logic is a two-valued logic: propositions in this logic are either true or false. In the first case, the truth value 1 is attributed to the proposition, while in the second case the attributed truth value is 0 . Given the truth values of two propositions $p$ and $q$, it is possible to derive the truth values of the negation 'not $p$ ' (and 'not $q$ '), the conjunction ' $p$ and $q$ ', the disjunction ' $p$ or $q$ ' and the implication ' $p$ implies

Bart Van Gasse,

Department of Applied Mathematics and Computer Science, Ghent University, Krijgslaan 281

(S9), B-9000 Gent, Belgium e-mail: Bart.VanGasse@UGent.be

Chris Cornelis,

Department of Applied Mathematics and Computer Science, Ghent University, Krijgslaan 281 (S9), B-9000 Gent, Belgium e-mail: Chris.Cornelis@UGent.be

Glad Deschrijver,

Department of Applied Mathematics and Computer Science, Ghent University, Krijgslaan 281

(S9), B-9000 Gent, Belgium e-mail: Glad.Deschrijver@UGent.be 
$q$ '. These formulas are denoted as $\neg p, p \& q, p \vee q$ and $p \rightarrow q$. The truth values are calculated using the operations ${ }^{1} \neg, *, \sqcup$ and $\Rightarrow$. The truth tables of these operations are given in Table 1.

Table 1 Truth tables of the operations in classical logic.

\begin{tabular}{llllll}
\hline$x$ & $y$ & $\neg x$ & $x * y$ & $x \sqcup y$ & $x \Rightarrow y$ \\
\hline 0 & 0 & 1 & 0 & 0 & 1 \\
0 & 1 & 1 & 0 & 1 & 1 \\
1 & 0 & 0 & 0 & 1 & 0 \\
1 & 1 & 0 & 1 & 1 & 1 \\
\hline
\end{tabular}

For more complicated formulas the truth values can be computed in the same way. For example, if $p$ is true and $q$ is false, then the truth value of $(p \vee q) \rightarrow((p \rightarrow$ $q) \rightarrow q)$ is calculated as follows: the truth value of $p \rightarrow q$ is $1 \Rightarrow 0=0$, so the truth value of $(p \rightarrow q) \rightarrow q$ is $0 \Rightarrow 0=1$. The truth value of $p \vee q$ is $1 \sqcup 0=1$. So we conclude that the truth value of $(p \vee q) \rightarrow((p \rightarrow q) \rightarrow q)$ is $1 \Rightarrow 1=1$. Interestingly, the truth value of this formula is always 1 , even if other truth values are attributed to $p$ and $q$. Such formulas are called tautologies. If a formula $\phi$ is a tautology, this is denoted as $\models \phi$. More generally, for a set of formulas $\Gamma, \Gamma \models \phi$ means "no matter what truth values are attributed to the propositions, if the truth values of the formulas in $\Gamma$ are 1 , then the truth value of $\phi$ is 1 ".

The two values 0 and 1, together with the defined operations, form a Boolean algebra. Therefore we say that this Boolean algebra is the semantics of classical logic. Saying that $(p \vee q) \rightarrow((p \rightarrow q) \rightarrow q)$ is a tautology in classical logic, is the same as saying that $(x \sqcup y) \Rightarrow((x \Rightarrow y) \Rightarrow y)=1$ is an identity in this Boolean algebra (meaning "whatever value of the Boolean algebra we give to $x$ and $y$, the calculation of $(x \sqcup y) \Rightarrow((x \Rightarrow y) \Rightarrow y)$ yields 1"). Now, identities in this Boolean algebra are also identities in every other Boolean algebra (we say that this Boolean algebra generates all Boolean algebras). Therefore classical logic does not only have the Boolean algebra with two elements as semantics, but also the whole variety of Boolean algebras: the general semantics of classical logic consist of all Boolean algebras.

Interestingly it is also possible to describe classical logic without using semantics. This is done with axioms and deduction rules, which allow to prove a formula from a set of formulas. When a formula $\phi$ is provable from a theory $\Gamma$, this is denoted as $\Gamma \vdash \phi$. Two important results in classical logic are soundness (if $\Gamma \vdash \phi$, then $\Gamma \models \phi$ ) and completeness (if $\Gamma \models \phi$, then $\Gamma \vdash \phi$ ). We write this shortly as $\Gamma \vdash \phi$ iff $\Gamma \models \phi$.

\footnotetext{
${ }^{1}$ Note that we use different symbols, to distinguish the logical connectives from the corresponding operations. Only for the negation we employ the same symbol.
} 
Now, for the truth values of several propositions one might prefer more than the two options 0 (false) and 1 (true). Indeed, for vague propositions like 'it is raining hard', it would be useful if one could attribute an intermediate truth value, somewhere between 'false' and 'true'. This can be done using fuzzy set theory, in which every element of the unit interval $[0,1]$ serves as a truth value, instead of only 0 and 1. The operations for the negation, conjunction, disjunction and implication were generalized to this setting. Later the structure of the unit interval was generalized to an arbitrary bounded lattice to allow for incomparabilities among elements, and triangular norms and conorms are quite common nowadays as generalized representations of logical conjunction and disjunction, respectively. An interesting class of these generalizations, especially from the logical point of view, are MTL-algebras [16]. In these structures, the operations modelling (strong) conjunction and implication are connected by the residuation principle. These MTL-algebras form the general semantics of monoidal t-norm based logic (MTL, [16]), in the same way Boolean algebras form the general semantics of classical logic. Similarly as for classical logic, these general semantics can be restricted. Indeed, MTL is also sound and complete w.r.t. standard MTL-algebras, i.e., MTL-algebras on the unit interval. Therefore MTL is called a (formal) fuzzy logic. But it is definitely not the only fuzzy logic. Indeed, by adding more axioms and/or deduction rules to the axioms and deduction rule of MTL, we obtain other fuzzy logics. It is even possible to retrieve classical logic in this way. Semantically speaking, this means that Boolean algebras are special cases of MTL-algebras. Some well-known fuzzy logics, situated between MTL and classical logic, are Hájek's Basic Logic (BL) [24], Łukasiewicz Logic (Ł) [30] and Gödel Logic (GL) [14, 22]. Also Intuitionistic Logic (IL) [25] can be seen as a fuzzy logic. These logics are sound and complete w.r.t. BL-algebras, MV-algebras (or, equivalently, Wajsberg algebras [20]), G-algebras and Heyting algebras, respectively. We refer to [17] for a comprehensive overview of these and other logics. Other general references on fuzzy logics are [6, 23, 24].

In [43], Zadeh introduced type-2 fuzzy sets, a generalization of fuzzy sets. The idea behind these structures is that they provide a way to express incomplete as well as graded knowledge; as opposed to fuzzy sets, which only express gradedness, not incompleteness. Unfortunately, type-2 fuzzy sets are quite complicated to work with. Therefore often interval-valued fuzzy sets are used. These special cases of type-2 fuzzy sets are easier to handle. Indeed, truth values in this setting are closed subintervals of the unit interval, and such an interval is determined by just two values: its lower and upper bound. The aim of this chapter is to develop a logic that has intervals as truth values. The intended semantics are residuated lattices on the set of closed subintervals of the unit interval. We call this set the triangularization of the unit interval. A particular subset of this triangularization is its so-called diagonal, consisting of those intervals for which the lower and upper bound coincide. These intervals are called exact intervals and represent truth values of propositions about which the knowledge is complete. Intuitively, the truth values of formulas constructed with these propositions should be exact intervals as well (because in these cases, the situation is similar to working with formulas in fuzzy 
logics). The semantics of so-called interval-valued fuzzy logics have already been examined by different authors. Especially interval-valued triangular norms, triangular conorms and implicators have received ample attention. Most of these authors $[1,2,15,21,26]$ only consider interval-valued operations that map the diagonal on the diagonal, although the most general definitions of triangular norms, triangular conorms and implicators allow other operations as well [7, 10, 11, 28]. Generally speaking, interval-valued operations do not satisfy as many properties as operations on the unit interval. For example, standard interval-valued residuated lattices can never satisfy prelinearity [8]. A lot of other properties can hold though. There are even interval-valued implicators that satisfy all the Smets-Magrez axioms [9].

The three main sections of this chapter are conceived as follows:

- In Section 2 we elaborate the theory of interval-valued residuated lattices, which include the intended semantics of interval-valued fuzzy logics.

- In Section 3 we give the definition of triangle algebras, which are algebraic structures describing interval-valued residuated lattices.

- In Section 4 we then introduce several interval-valued fuzzy logics and examine their properties, in particular the soundness and completeness w.r.t. the intended and the general semantics.

Before we continue, we recall some algebraic concepts that will be used in this chapter.

- An algebra of type $\left(n_{1}, n_{2}, \ldots, n_{m}\right)$, with $n_{1}, n_{2}, \ldots, n_{m}$ non-negative integers, is a structure $\left(A, f_{1}, \ldots, f_{m}\right)$ in which $A$ is a set, $f_{1}$ an $n_{1}$-ary operation on $A, \ldots$ and $f_{m}$ an $n_{m}$-ary operation on $A$. If $n_{i}$ is 0 , then $f_{i}$ is a constant.

- A reduct of an algebra is an algebra on the same set, but in which some of the operations are left out. An algebra $\mathscr{A}$ is an expansion of an algebra $\mathscr{B}$ if $\mathscr{B}$ is a reduct of $\mathscr{A}$.

- A subalgebra of an algebra $\mathscr{A}=\left(A, f_{1}, \ldots, f_{m}\right)$ is an algebra on a subset $A^{\prime}$ of $A$ in which all operations of $\mathscr{A}$ are restricted to $A^{\prime}$. Of course, this is only possible if $A^{\prime}$ is closed under all these operations, i.e., if for every operation $f_{i}$ of $\mathscr{A}$, $f_{i}\left(a_{1}, \ldots, a_{n}\right) \in A^{\prime}$ whenever the arguments $a_{1}, \ldots, a_{n}$ are in $A^{\prime}$ (with $n$ the arity of $\left.f_{i}\right)$.

- A morphism from an algebra $\mathscr{A}=\left(A, f_{1}, \ldots, f_{m}\right)$ to an algebra $\mathscr{B}=\left(B, g_{1}, \ldots\right.$, $\left.g_{m}\right)$ of the same type, is a mapping $h$ from $A$ to $B$ such that $h\left(f_{i}\left(a_{1}, \ldots, a_{n}\right)\right)=$ $g_{i}\left(h\left(a_{1}\right), \ldots, h\left(a_{n}\right)\right)$ for all operations $f_{i}$ of $\mathscr{A}$ and all $a_{1}, \ldots, a_{n}$ in $A$ (with $n$ the arity of $f_{i}$ ).

- An embedding of an algebra $\mathscr{A}=\left(A, f_{1}, \ldots, f_{m}\right)$ in an algebra $\mathscr{B}=\left(B, g_{1}, \ldots\right.$, $\left.g_{m}\right)$ of the same type, is a morphism $h$ from $A$ to $B$ such that $h\left(a_{1}\right) \neq h\left(a_{2}\right)$ whenever $a_{1} \neq a_{2}$.

- An isomorphism from an algebra $\mathscr{A}=\left(A, f_{1}, \ldots, f_{m}\right)$ to an algebra $\mathscr{B}=(B$, $\left.g_{1}, \ldots, g_{m}\right)$ of the same type, is an embedding of $A$ in $B$ such that for every element $b$ of $B, b=h(a)$ for some $a$ in $A$. 


\section{Interval-Valued Structures}

The most general semantics of fuzzy logics do not only contain algebraic structures on the unit interval, it consists of all residuated lattices. For interval-valued fuzzy logics, the situation is comparable: the most general semantics are interval-valued residuated lattices. In this section, we propose a definition of these structures.

\subsection{Triangularizations of Partially Ordered Sets}

Definition 1. [39] Given any partially ordered set (shortly: poset) $\mathscr{P}=(P, \leq)$, we can define its triangularization $\mathbb{T}(\mathscr{P})=(\operatorname{Int}(\mathscr{P}), \preceq)$ in the following way:

- $\operatorname{Int}(\mathscr{P})=\left\{\left[p_{1}, p_{2}\right] \mid\left(p_{1}, p_{2}\right) \in P^{2}\right.$ and $\left.p_{1} \leq p_{2}\right\}$,

- $\left[p_{1}, p_{2}\right] \preceq\left[q_{1}, q_{2}\right]$ iff $p_{1} \leq q_{1}$ and $p_{2} \leq q_{2}$, for all $\left[p_{1}, p_{2}\right]$ and $\left[q_{1}, q_{2}\right]$ in $\operatorname{Int}(\mathscr{P})$.

The elements of $\operatorname{Int}(\mathscr{P})$ are called the intervals of $\mathscr{P}$.

The first and the second projection $\mathrm{pr}_{1}$ and $\mathrm{pr}_{2}$ are the mappings from $\operatorname{Int}(\mathscr{P})$ to $P$, defined by $\operatorname{pr}_{1}\left(\left[x_{1}, x_{2}\right]\right)=x_{1}$ and $\operatorname{pr}_{2}\left(\left[x_{1}, x_{2}\right]\right)=x_{2}$, for all $\left[x_{1}, x_{2}\right]$ in $\operatorname{Int}(\mathscr{P})$.

The vertical and the horizontal projection $\mathrm{pr}_{v}$ and $\mathrm{pr}_{h}$ are the mappings from $\operatorname{Int}(\mathscr{P})$ to $\operatorname{Int}(\mathscr{P})$, defined by $\operatorname{pr}_{v}\left(\left[x_{1}, x_{2}\right]\right)=\left[x_{1}, x_{1}\right]$ and $\operatorname{pr}_{h}\left(\left[x_{1}, x_{2}\right]\right)=\left[x_{2}, x_{2}\right]$, for all $\left[x_{1}, x_{2}\right]$ in $\operatorname{Int}(\mathscr{P})$.

It is straightforward to verify that for any poset $\mathscr{P}, \mathbb{T}(\mathscr{P})$ is also a poset. Moreover, the original poset $(P, \leq)$ is contained in $\mathbb{T}(\mathscr{P})$ in some way: indeed, the mapping $i: P \rightarrow \operatorname{Int}(\mathscr{P})$ defined by $i(p)=[p, p]$ for all $p$ in $P$, is injective and preserves the ordering (if $p \leq q$, then $i(p)=[p, p] \preceq[q, q]=i(q)$ ). The image $i(P)$ consists of the intervals $\left[p_{1}, p_{2}\right]$ in $\operatorname{Int}(\mathscr{P})$ for which $p_{1}=p_{2}$. The elements of $i(P)$ are called exact intervals. The subset $i(P)$ of $\operatorname{Int}(\mathscr{P})$ is often referred to as the diagonal of $\mathbb{T}(\mathscr{P})$. Note that $\mathrm{pr}_{v}=i \circ \mathrm{pr}_{1}$ and $\mathrm{pr}_{h}=i \circ \mathrm{pr}_{2}$, and that $i(P)=\operatorname{pr}_{v}(\operatorname{Int}(\mathscr{P}))=\operatorname{pr}_{h}(\operatorname{Int}(\mathscr{P}))$.

Example 1. The poset that will be of central interest in this chapter is $\mathbb{T}([0,1], \leq)$ : the closed subintervals of the unit interval. This poset is complete and its order is not linear. Its graphical representation as a triangle is shown in Figure 1. The diagonal is the hypothenuse of this triangle. Note that the shape of this representation is triangular. This holds for all triangularizations of bounded linear posets, hence the name 'triangularization'.

\subsection{Triangular Lattices}

Recall that a lattice is a poset in which the supremum and infimum of every two elements exist; on the other hand, often the following equivalent definition is also used. 


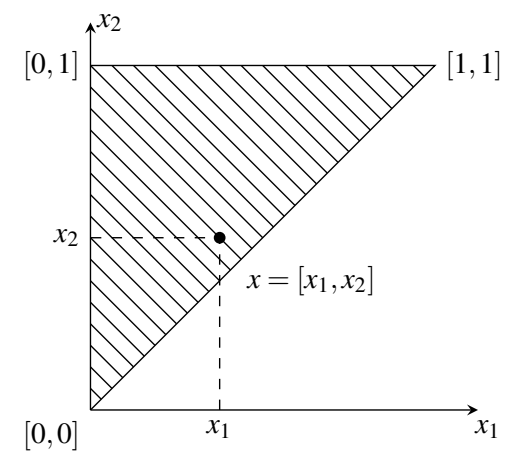

Fig. 1 The lattice $\mathbb{T}([0,1], \leq)$

Definition 2. A lattice is an algebra $(L, \sqcap, \sqcup)$ of type $(2,2)$ such that $\sqcap$ ('meet') and $\sqcup$ ('join') are idempotent, commutative and associative operations satisfying the following absorption laws: for all $x$ and $y$ in $L, x \sqcup(x \sqcap y)=x$ and $x \sqcap(x \sqcup y)=x$. The lattice order $\leq$ is defined by $x \leq y$ iff $x \sqcap y=x$ (or, equivalently, iff $x \sqcup y=y$ ), for all $x$ and $y$ in $L$.

Because lattices can be seen as posets, we can consider their triangularizations. One easily observes that the infimum (resp. supremum) on the triangularization of a lattice is obtained by taking the infimum (resp. supremum) of the first and second projections. More precisely: for any lattice $\mathscr{L}=(L, \sqcap, \sqcup)$, the infimum $\prod$ and supremum $\sqcup$ on its triangularization $\mathbb{T}(\mathscr{L})$ are given by

- $\left[x_{1}, x_{2}\right] \sqcap\left[y_{1}, y_{2}\right]=\left[x_{1} \sqcap y_{1}, x_{2} \sqcap y_{2}\right]$,

- $\left[x_{1}, x_{2}\right] \sqcup\left[y_{1}, y_{2}\right]=\left[x_{1} \sqcup y_{1}, x_{2} \sqcup y_{2}\right]$,

for all $\left[x_{1}, x_{2}\right]$ and $\left[y_{1}, y_{2}\right]$ in $\operatorname{Int}(\mathscr{L})$. Note that we use big $П$ - and $\sqcup$-symbols for the intervals of the triangularization, and small $\sqcap$ - and $\sqcup$-symbols for the elements of the original lattice.

It can be verified that $\mathbb{T}(\mathscr{L})$ is a lattice iff $\mathscr{L}$ is a lattice, that $i$ (as defined in Section 2.1) is a morphism from $(L, \sqcap, \sqcup)$ to $(\operatorname{Int}(\mathscr{L}), \sqcap, \sqcup)$, and that the set $i(L)$ of exact intervals is therefore closed under $\prod$ and $\bigsqcup$ and forms a sublattice $\mathscr{E}(\mathscr{L})=$ $(i(L), \sqcap, \sqcup)$.

On the other hand, $\mathbb{T}(\mathscr{L})$ is bounded iff $\mathscr{L}$ is bounded. In this case, the smallest (resp. greatest) element of $\mathscr{L}$ is usually denoted by 0 (resp. 1), and the smallest (resp. greatest) element of $\mathbb{T}(\mathscr{L})$ by $[0,0]$ (resp. [1,1]). As we will see later on, the element $[0,1]$ will also play an important role, along with the projections $\operatorname{pr}_{v}$ and $\mathrm{pr}_{h}$.

For any triangularization $(\operatorname{Int}(\mathscr{L}), \sqcap, \sqcup)$ of a bounded lattice $\mathscr{L}=(L, \sqcap, \sqcup)$ (with smallest element 0 and greatest element 1$)$, we call $\left(\operatorname{Int}(\mathscr{L}), \sqcap, \sqcup, \mathrm{pr}_{v}, \mathrm{pr}_{h}\right.$, $[0,0],[0,1],[1,1])$ the extended triangularization of $\mathscr{L}$. Below, we show that extended triangularizations can be captured by a class of algebraic structures defined only with identities: the variety of triangular lattices. 
Definition 3. [36] A triangular lattice ${ }^{2}$ is an algebra $(L, \sqcap, \sqcup, v, \mu, 0, u, 1)$ of type $(2,2,1,1,0,0,0)$ such that $(L, \sqcap, \sqcup)$ is a bounded lattice with smallest element 0 and greatest element 1 such that
(T.1) $v x \leq x$,
(T.2) $v x \leq v v x$
$\left(T .1^{\prime}\right) \quad x \leq \mu x$
(T.3) $v(x \sqcap y)=v x \sqcap v y$,
$\left(T .2^{\prime}\right) \quad \mu \mu x \leq \mu x$
(T.4) $v(x \sqcup y)=v x \sqcup v y$,
$\left(T .3^{\prime}\right) \quad \mu(x \sqcap y)=\mu x \sqcap \mu y$,
(T.5) $v u=0$
$\left(T .4^{\prime}\right) \quad \mu(x \sqcup y)=\mu x \sqcup \mu y$,
(T.6) $v \mu x=\mu x$
(T.5') $\mu u=1$,
(T.10) $x=v x \sqcup(\mu x \sqcap u)$,
$\left(T .6^{\prime}\right) \mu v x=v x$
$\left(T .10^{\prime}\right) x=\mu x \sqcap(v x \sqcup u)$.

The unary operators $v$ and $\mu$ are called the necessity and possibility operator, respectively.

Note that from (T.10) it follows that for all $x$ and $y$ in $L$,

$$
x=y \text { whenever } v x=v y \text { and } \mu x=\mu y .
$$

From (T.1) and (T.2), it is clear that in a triangular lattice, always $v v x=v x$. Similarly, $\mu \mu x=\mu x$. Each of (T.3) and (T.4) implies that $v$ is an increasing operator. In the same way, (T.3') or (T.4') force $\mu$ to be increasing too. Other properties that follow easily are $v 1=1$ and $\mu 0=0: v 1=v \mu u=\mu u=1$ and $\mu 0=\mu v u=v u=0$. Together with (T.1), (T.1'), (T.2), (T.2'), (T.3) and (T.4'), they mean that $\mu$ is a closure operator, and $v$ is an interior operator. Both are also lattice morphisms.

Note that (T.1')-(T.4') are conditions for $\mu$, which is similar to the modal possibility operator; they are dual to (T.1)-(T.4) for $v$, which is similar to the modal necessity operator. Only (T.4) and (T.3') are different: in the modal setting, they are in general not true; and one doesn't want them to be true either (see e.g. [44]). In general, we do not require dependency of $\mu$ on $v$. The conditions (T.5) and (T.5') express the complete lack of knowledge about $u$ : its necessity is 0 , but its possibility is 1 . The conditions (T.6) and (T.6') are known in modal logics as the S5-principles [31, 32].

Proposition 1. [36] Let $(L, \sqcap, \sqcup, v, \mu, 0, u, 1)$ be a triangular lattice. Then $(L, \sqcap, \sqcup$, $v, \mu, 0, u, 1)$ is isomorphic to the extended triangularization of a bounded lattice. Conversely, every extended triangularization of a bounded lattice is a triangular lattice.

In the remainder, we will use the set of exact elements $E(\mathscr{L})$ of a triangular lattice $\mathscr{L}=(L, \sqcap, \sqcup, v, \mu, 0, u, 1)$ defined by $\{x \in L \mid v x=x\}$. It is closed under all the defined (unary and binary) operators, and therefore $\mathscr{E}(\mathscr{L})=(E(\mathscr{L}), \sqcap, \sqcup)$ (in which the binary operators are restricted to $E(\mathscr{L}))$ is a bounded lattice.

\footnotetext{
${ }^{2}$ The reason we call the last two conditions (T.10) and (T.10') instead of (T.7) and (T.7') is that we would like to keep the same notations as in the papers [36, 37, 38, 40, 42].
} 


\subsection{Interval-Valued Residuated lattices}

For most formal fuzzy logics the semantics require not only a partial order on the set of truth values, but also some extra operations that model 'AND' (the strong conjunction - the infimum being the weak conjunction) and 'IMPLIES' (the implication). A very commonly used structure - and also the basic structure that we will use in this work - is that of residuated lattices.

Definition 4. A residuated lattice ${ }^{3}$ is a structure $\mathscr{L}=(L, \sqcap, \sqcup, *, \Rightarrow, 0,1)$ in which $\sqcap, \sqcup, *$ and $\Rightarrow$ are binary operators on the set $L$ and

- $(L, \sqcap, \sqcup)$ is a bounded lattice with 0 as smallest and 1 as greatest element,

- $*$ is commutative and associative, with 1 as neutral element, and

- $x * y \leq z$ iff $x \leq y \Rightarrow z$ for all $x, y$ and $z$ in $L$ (residuation principle).

The binary operations $*$ and $\Rightarrow$ are called product and implication, respectively. We will use the notations $\neg x$ for $x \Rightarrow 0$ (negation), $x \Leftrightarrow y$ for $(x \Rightarrow y) \sqcap(y \Rightarrow x)$.

The following kinds of residuated lattices are used in this chapter.

\section{Definition 5.}

- An MTL-algebra [16] is a prelinear residuated lattice, i.e., a residuated lattice in which $(x \Rightarrow y) \sqcup(y \Rightarrow x)=1$ for all $x$ and $y$ in $L$.

- A BL-algebra [24] is a divisible MTL-algebra, i.e., an MTL-algebra in which $x \sqcap y=x *(x \Rightarrow y)$ for all $x$ and $y$ in $L$. The weaker property $x \sqcap y=(x *(x \Rightarrow$ $y)) \sqcup(y *(y \Rightarrow x))$ is called weak divisibility.

- An MV-algebra [3, 4] is a BL-algebra in which the negation is an involution, i.e., $(x \Rightarrow 0) \Rightarrow 0=x$ for all $x$ in $L$.

- A Boolean algebra [27] is an MV-algebra that is also a Heyting-algebra, i.e., in which $x * x=x$ for all $x$ in $L$, or, equivalently, in which $*=\sqcap$.

In a residuated lattice, the operator $*$ is always a residuated t-norm, with $\Rightarrow$ as its residual implicator ${ }^{4}$. Conversely, if $T$ is a residuated t-norm on a bounded lattice $(L, \sqcap, \sqcup)$, then $\left(L, \sqcap, \sqcup, T, I_{T}, 0,1\right)$ is a residuated lattice. Note however that not all t-norms are residuated. In complete lattices $(L, \sqcap, \sqcup)$, a t-norm $T$ is residuated (and therefore induces a residuated lattice) iff it satisfies $T(x, \sup Y)=\sup \{T(x, y) \mid y \in$ $Y\}$, for all $x$ in $L$ and $Y \subseteq L$ [35].

Example 2. Let $T$ be a t-norm on $([0,1], \min , \max )$. It is well-known (see, e.g., [17, 24] that

\footnotetext{
${ }^{3}$ In the literature (e.g. in [26]), the name residuated lattice is sometimes used for structures more general than what we call residuated lattices. In the most general terminology, our structures would be called bounded integral commutative residuated lattices.

${ }^{4}$ Recall that a triangular norm (t-norm, for short) on a poset $(P, \leq)$ with largest element 1 , is a binary, increasing, commutative and associative operator $T: P^{2} \rightarrow P$ that satisfies $T(x, 1)=1$, for all $x$ in $P$. If for every pair $(x, y)$ in $P^{2}, \sup \{z \in P \mid T(x, z) \leq y\}$ exists, then the map $I_{T}$ defined by $I_{T}(x, y)=\sup \{z \in P \mid T(x, z) \leq y\}$ is called the residual implicator of $T$. A t-norm $T$ is called residuated if it has a residual implicator satisfying $I_{T}(x, y)=\max \{z \in P \mid T(x, z) \leq y\}$, in other words if for any pair $(x, y)$ in $P^{2}$ the set $\{z \in P \mid T(x, z) \leq y\}$ has a maximum.
} 
- $T$ is residuated iff $T$ is left-continuous,

- $\left([0,1], \min , \max , T, I_{T}, 0,1\right)$ is an MTL-algebra ${ }^{5}$ iff $T$ is left-continuous,

- $\left([0,1], \min , \max , T, I_{T}, 0,1\right)$ is a BL-algebra iff $T$ is continuous,

- ([0,1], min, max, $\left.T, I_{T}, 0,1\right)$ is an MV-algebra iff $T$ is conjugated to the Eukasiewicz t-norm $T_{W}$, i.e., iff there exists a strictly increasing bijection $\phi:[0,1] \rightarrow$ $[0,1]$ such that $T(x, y)=\phi^{-1}\left(T_{W}(\phi(x), \phi(y))\right)$, where $T_{W}(x, y)=\max (0, x+y-$ $1)$.

Several t-norms can be defined on triangularizations of bounded lattices. A class that will be of great importance later on, consists of t-norms constructed from a tnorm on and an element of the original lattice: if $T$ is a t-norm on a lattice $\mathscr{L}=$ $(L, \sqcap, \sqcup)$ which has a greatest element 1 , and $t$ is an element of $L$, then $\mathscr{T}_{T, t}$, defined by

$$
\mathscr{T}_{T, t}\left(\left[x_{1}, x_{2}\right],\left[y_{1}, y_{2}\right]\right)=\left[T\left(x_{1}, y_{1}\right), T\left(T\left(x_{2}, y_{2}\right), t\right) \sqcup T\left(x_{1}, y_{2}\right) \sqcup T\left(x_{2}, y_{1}\right)\right],
$$

for all $\left[x_{1}, x_{2}\right]$ and $\left[y_{1}, y_{2}\right]$ in $\operatorname{Int}(\mathscr{L})$, is a t-norm on $\mathbb{T}(\mathscr{L})$. These t-norms were introduced by Deschrijver and Kerre in [11].

Recall that we are working towards a variety of algebraic structures suitable as semantics for a logic with intervals as truth values. At this point it might seem a good idea to choose residuated lattices on triangularizations (or, equivalently, residuated lattices on triangular lattices). However, in these structures the set of exact intervals is not necessarily closed under the product and implication. This counters the intuition that the truth values of the propositions $p \& q$ and $p \rightarrow q$ should be exact if the truth values of $p$ and $q$ are exact. Therefore, residuated lattices on triangularizations are too general to serve as the desired semantics. This leads us to the definition of interval-valued residuated lattices.

Definition 6. [41]

- An interval-valued residuated lattice (IVRL) is a residuated lattice $(\operatorname{Int}(\mathscr{L}), \sqcap$, $\sqcup, *, \Rightarrow,[0,0],[1,1])$ on the triangularization $\mathbb{T}(\mathscr{L})$ of a bounded lattice $\mathscr{L}=$ $(L, \sqcap, \sqcup)$, in which the diagonal $i(L)$ is closed under $*$ and $\Rightarrow$, i.e., $\left[x_{1}, x_{1}\right] *$ $\left[y_{1}, y_{1}\right] \in i(L)$ (with $i$ the injection defined in Section 2.1) and $\left[x_{1}, x_{1}\right] \Rightarrow\left[y_{1}, y_{1}\right] \in$ $i(L)$ for all $x_{1}$ and $y_{1}$ in $L$.

- When we add $[0,1]$ as a constant, and $\operatorname{pr}_{v}$ and $\operatorname{pr}_{h}$ (as defined in Section 2.1) as unary operators, the structure $\left(\operatorname{Int}(\mathscr{L}), \sqcap, \sqcup, *, \Rightarrow, \operatorname{pr}_{v}, \operatorname{pr}_{h},[0,0],[0,1],[1,1]\right)$ is called an extended IVRL.

An IVRL in which $\mathscr{L}=([0,1], \min , \max )$ is called a standard IVRL. An extended IVRL in which $\mathscr{L}=([0,1], \min , \max )$ is called a standard extended IVRL.

By Proposition 1, if $\left(\operatorname{Int}(\mathscr{L}), \sqcap, \sqcup, *, \Rightarrow, \operatorname{pr}_{v}, \operatorname{pr}_{h},[0,0],[0,1],[1,1]\right)$ is an extended IVRL, then it is also a triangular lattice. Now we show that the extra operations $*$ and $\Rightarrow$ satisfy the following two properties, for all $x$ and $y$ in $\operatorname{Int}(\mathscr{L})$ :

${ }^{5}$ Because $([0,1], \min , \max )$ is linear, every residuated lattice on this lattice is automatically an MTL-algebra. 
- $\operatorname{pr}_{v}(x) * \operatorname{pr}_{v}(y) \leq \operatorname{pr}_{v}\left(\operatorname{pr}_{v}(x) * \operatorname{pr}_{v}(y)\right)$,

- $\operatorname{pr}_{v}(x) \Rightarrow \operatorname{pr}_{v}(y) \leq \operatorname{pr}_{v}\left(\operatorname{pr}_{v}(x) \Rightarrow \operatorname{pr}_{v}(y)\right)$.

Indeed, the first property is equivalent to $\operatorname{pr}_{v}(x) * \operatorname{pr}_{v}(y)=\operatorname{pr}_{v}\left(\operatorname{pr}_{v}(x) * \operatorname{pr}_{v}(y)\right)$, which means that for any $a$ and $b$ in $i(L), a * b=\operatorname{pr}_{v}(a * b)$, in other words $a * b \in i(L)$. So it tells us exactly that the diagonal $i(L)$ is closed under $*$. And similarly, the second property means the diagonal $i(L)$ is closed under $\Rightarrow$ too.

These two properties suggest a way to describe these IVRLs, which seem suitable as semantics for interval-valued fuzzy logic, using only identities. This leads us to the next section, where we will introduce this variety (called triangle algebras) and study its properties in detail.

\section{Triangle algebras}

In this section, we introduce a variety of structures called triangle algebras, and show that they are isomorphic to extended IVRLs. Then, we investigate the product and implication of triangle algebras and show that they are determined by their action on the exact elements and by one specific product: $u * u$. This characterization is used to uncover the connections between properties on triangle algebras and properties on their subalgebras of exact elements.

\subsection{Definition and Connection with IVRLs}

In the definition of a triangle algebra we want to combine the structure of a residuated lattice and the structure of intervals (equipped with the order in Definition 1), plus the desired property that the subset of exact intervals is closed under all defined operations. This leads us to the following definition.

Definition 7. [41, 36] A triangle algebra is a structure $(A, \sqcap, \sqcup, *, \Rightarrow, v, \mu, 0, u, 1)$ of type $(2,2,2,2,1,1,0,0,0)$ such that $(A, \sqcap, \sqcup, v, \mu, 0, u, 1)$ is a triangular lattice, $(A, \sqcap, \sqcup, *, \Rightarrow, 0,1)$ is a residuated lattice, and satisfying for all $x$ and $y$ in $A$,

$\left(T .7^{\prime}\right) v x * v y \leq v(v x * v y)$,

(T.9) $v x \Rightarrow v y \leq v(v x \Rightarrow v y)$.

In other words, a structure $(A, \sqcap, \sqcup, *, \Rightarrow, v, \mu, 0, u, 1)$ of type $(2,2,2,2,1,1,0,0,0)$ such that $(A, \sqcap, \sqcup, *, \Rightarrow, 0,1)$ is a residuated lattice, and satisfying for all $x$ and $y$ in A, 


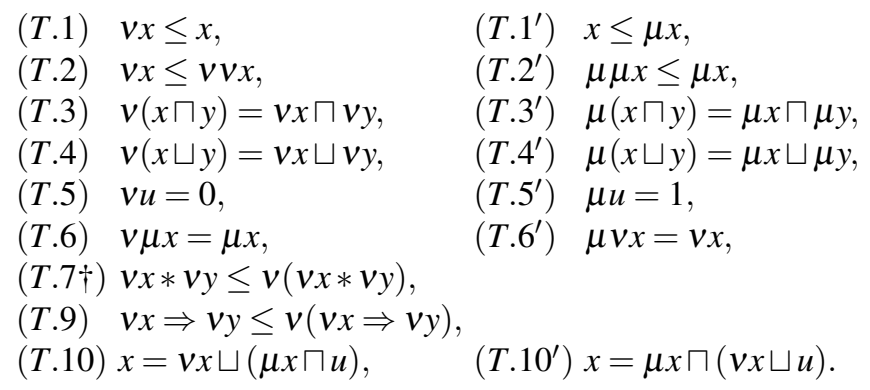

A triangle algebra $\left(A, \sqcap, \sqcup, *, \Rightarrow, v, \mu, 0_{A}, u_{A}, 1_{A}\right)$ is called a standard triangle algebra iff $(A, \sqcap, \sqcup)=\mathbb{T}([0,1], \min , \max )$.

In a standard triangle algebra $\left(A, \sqcap, \sqcup, *, \Rightarrow, v, \mu, 0_{A}, u_{A}, 1_{A}\right)$ it holds that $0_{A}=[0,0]$, $1_{A}=[1,1], u=[0,1], v\left[x_{1}, x_{2}\right]=\left[x_{1}, x_{1}\right]$ and $\mu\left[x_{1}, x_{2}\right]=\left[x_{2}, x_{2}\right]$ for all $\left[x_{1}, x_{2}\right]$ in $\operatorname{Int}([0,1], \min , \max )[41]$. Because a triangle algebra is an expansion of both a triangular lattice and a residuated lattice, the properties of these kinds of structures remain valid in triangle algebras. The connections between triangle algebras and several related algebraic structures from the literature are studied extensively in $[39,41,36]$. In this chapter, we focus on the relationship with IVRLs.

Proposition 2. [41] Let $\left(\operatorname{Int}(\mathscr{L}), \sqcap, \sqcup, *, \Rightarrow, \mathrm{pr}_{v}, \mathrm{pr}_{h},[0,0],[0,1],[1,1]\right)$ be an extended IVRL. Then $\left(\operatorname{Int}(\mathscr{L}), \sqcap, \sqcup, *, \Rightarrow, \mathrm{pr}_{v}, \mathrm{pr}_{h},[0,0],[0,1],[1,1]\right)$ is a triangle algebra. Conversely, let $\mathscr{A}=(A, \sqcap, \sqcup, *, \Rightarrow, v, \mu, 0, u, 1)$ be a triangle algebra. Then $\mathscr{A}$ is isomorphic to an extended IVRL.

The isomorphism $\chi$ that is used in the proof of Proposition 2 is depicted graphically in Figure 2.

\subsection{Characterization of Product and Implication. Decomposition Theorem.}

The following important proposition reveals that the implication $\Rightarrow$ and the product $*$ are completely determined by their action on the diagonal and the value of $u * u$ :

Proposition 3. [40] In a triangle algebra $\mathscr{A}=(A, \sqcap, \sqcup, *, \Rightarrow, v, \mu, 0, u, 1)$, it holds that

- $v(x \Rightarrow y)=(v x \Rightarrow v y) \sqcap(\mu x \Rightarrow \mu y)$,

- $\mu(x \Rightarrow y)=(\mu x \Rightarrow(\mu(u * u) \Rightarrow \mu y)) \sqcap(v x \Rightarrow \mu y)$,

- $v(x * y)=v x * v y$,

- $\mu(x * y)=(v x * \mu y) \sqcup(\mu x * v y) \sqcup(\mu x * \mu y * \mu(u * u))$

and therefore (by (T.10) and (T.10')) 


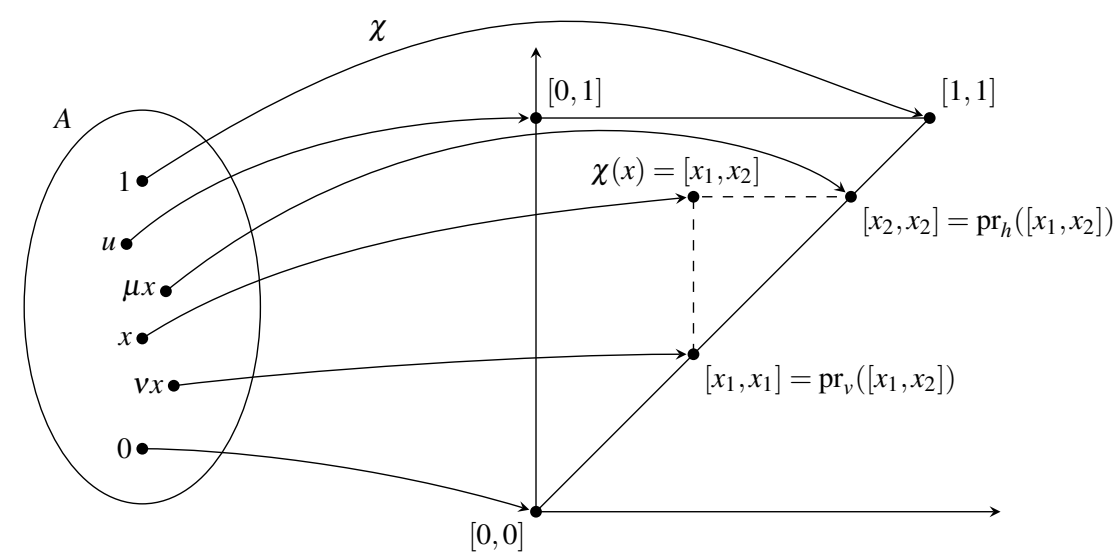

Triangle algebra

$(A, \sqcap, \sqcup, *, \Rightarrow, v, \mu, 0, u, 1)$

Isomorphic extended IVRL

$\left(A^{\prime}, \sqcap^{\prime}, \sqcup^{\prime}, *^{\prime}, \Rightarrow^{\prime}, \operatorname{pr}_{v}, \operatorname{pr}_{h},[0,0],[0,1],[1,1]\right)$

Fig. 2 The isomorphism $\chi$ from a triangle algebra to an extended IVRL.

$$
\begin{aligned}
& x \Rightarrow y \\
& =(((\mu x \Rightarrow(\mu(u * u) \Rightarrow \mu y)) \sqcap(v x \Rightarrow \mu y)) \sqcap u) \sqcup((\mu x \Rightarrow \mu y) \sqcap(v x \Rightarrow v y)) \\
& =(((\mu x \Rightarrow \mu y) \sqcap(v x \Rightarrow v y)) \sqcup u) \sqcap((\mu x \Rightarrow(\mu(u * u) \Rightarrow \mu y)) \sqcap(v x \Rightarrow \mu y))
\end{aligned}
$$

and

$$
\begin{aligned}
x * y & =(((v x * \mu y) \sqcup(\mu x * v y) \sqcup(\mu x * \mu y * \mu(u * u))) \sqcap u) \sqcup(v x * v y) \\
& =((v x * v y) \sqcup u) \sqcap((v x * \mu y) \sqcup(\mu x * v y) \sqcup(\mu x * \mu y * \mu(u * u))) .
\end{aligned}
$$

Because of Proposition 3, the product and implication in triangle algebras are always of a specific form, which implies that a triangle algebra is completely determined by its subalgebra of exact elements and the value $u * u$ (in triangle algebras, $u$ is a constant, playing the role of the interval [0,1] in IVRLs). Conversely, for a fixed residuated lattice $\mathscr{L}$ and element $\alpha$ in that lattice, we can construct a triangle algebra with $\mathscr{L}$ as subalgebra of exact elements and $u * u$ determined by $\mu(u * u)=\alpha$. So we can conclude that there is a one-to-one correspondence between triangle algebras and couples $(\mathscr{L}, \alpha)$, in which $\alpha$ is an element of the residuated lattice $\mathscr{L}$. 
This characterization implies that every property that can be imposed on triangle algebras, can be formulated in terms of such couples.

In particular, any property defined in Definition 5 can be weakened, by imposing it on $E(\mathscr{A})$ (instead of $\mathscr{A}$ ) only. We will denote this with the prefix 'pseudo'. For example, a triangle algebra is said to be pseudo-linear if its set of exact elements is linearly ordered (by the original ordering, restricted to the diagonal). Another example: a triangle algebra is pseudo-divisible if $v x \sqcap v y=v x *(v x \Rightarrow v y)$ for all $x$ and $y$ in $A(E(\mathscr{A})$ consists exactly of the elements of the form $v x)$.

It is well-known that MTL-algebras are isomorphic to subdirect products of linear residuated lattices $[16,24]$. This is a very useful result, as it implies that identities valid in all linear residuated lattices are also valid in all MTL-algebras, which significantly simplifies several proofs, and which is also needed for the chaincompleteness of the corresponding logic MTL (see Section 4). The 'interval-valued counterpart' of this result is given below:

Theorem 1. [42] Every pseudo-prelinear triangle algebra $\mathscr{A}$ is isomorphic to a subalgebra of the direct product of a system of pseudo-linear triangle algebras.

\section{Interval-valued fuzzy logics}

In Section 2 we have given the definition of interval-valued residuated lattices (IVRLs), and in Section 3 we have introduced triangle algebras to capture their structure by means of identities and/or inequalities. Using this characterization, we give the definition of several (propositional) interval-valued fuzzy logics in Section 4.2. But first, in Section 4.1 we give an overview of the well-studied common fuzzy logics, on which our interval-valued fuzzy logics are based, and mention their most important properties. In Section 4.3 we then investigate which of these properties hold for interval-valued fuzzy logics as well. In particular, we prove the soundness and completeness with respect to the algebraic semantics and the deduction theorem.

\subsection{Formal fuzzy logics}

Because in interval-valued fuzzy logics there will be more formulae, we make a distinction between formulae for fuzzy logics (FL-formulae) and formulae for intervalvalued fuzzy logics (IVFL-formulae).

Definition 8. FL-formulae are built up from a countable set of propositional variables (denoted by $p, q, r, p_{1}, p_{2}, \ldots$ ) and the constant $\overline{0}$. These symbols are FLformulae by definition. The other FL-formulae are defined recursively: if $\phi$ and $\psi$ are FL-formulae, then so are $(\phi \wedge \psi),(\phi \vee \psi),(\phi \& \psi)$ and $(\phi \rightarrow \psi)$.

The set of FL-formulae is denoted by $\mathscr{F}_{F L}$. 
In order to avoid unnecessary brackets, we agree on the following priority rules:

- among the connectives, \& has the highest priority; furthermore $\wedge$ and $\vee$ take precedence over $\rightarrow$,

- the outermost brackets are not written.

The following notations are used: $\overline{1}$ for $\overline{0} \rightarrow \overline{0}, \neg \phi$ for $\phi \rightarrow \overline{0}, \phi^{2}$ for $\phi \& \phi, \phi^{n}$ (with $n \in\{3,4,5, \ldots\}$ ) for $\left(\phi^{n-1}\right) \& \phi$ (moreover, $\phi^{0}$ is $\overline{1}$ and $\phi^{1}$ is $\phi$ ), and $\phi \leftrightarrow \psi$ for $(\phi \rightarrow \psi) \wedge(\psi \rightarrow \phi)$, for FL-formulae $\phi$ and $\psi$.

The FL-formulae $\phi \& \psi, \phi \rightarrow \psi$ and $\neg \phi$ stand for what we understand intuitively by ' $\phi$ and $\psi$ ' (strong conjunction), ' $\phi$ implies $\psi$ ' (implication) and 'not $\phi$ ' (negation).

It is impossible to list all true FL-formulae of a specific fuzzy logic, because their number is not finite. Therefore axioms and deduction rules are used. In the logics we deal with, an FL-formula is true if it is provable from the axioms using the deduction rules. We will explain this in more detail. This method also allows to prove FL-formulae from a given set of FL-formulae (usually called a theory). This means that in the proof of an FL-formula not only axioms of the logic can be used but also formulae of the theory. If an FL-formula $\phi$ is provable from a theory $\Gamma$ in a fuzzy logic $L$, this is denoted as $\Gamma \vdash_{L} \phi$. The relation $\vdash$ is called provability relation or syntactic consequence.

It is often not very easy to find out if an FL-formula is true in a specific fuzzy logic. A proof might be difficult to find and such a proof can become very long. This is why soundness and completeness of (fuzzy) logics is so important. It provides a way to determine if a formula is true or provable from a theory in a purely algebraic way. Indeed, soundness and completeness of a fuzzy logic are two properties relative to a class of algebraic structures. We call such a class a semantics of the fuzzy logic. To explain this connection between formal logic and algebra in more detail, we need some terminology first.

Definition 9. Let $\mathscr{L}=(L, \sqcap, \sqcup, *, \Rightarrow, 0,1)$ be a residuated lattice, $\Gamma$ a theory (i.e., a set of FL-formulae). An $\mathscr{L}$-evaluation is a mapping $e$ from the set of FL-formulae ${ }^{6}$ to $L$ that satisfies, for each two formulae $\phi$ and $\psi$ :

- $e(\phi \wedge \psi)=e(\phi) \sqcap e(\psi)$,

- $e(\phi \vee \psi)=e(\phi) \sqcup e(\psi)$,

- $e(\phi \& \psi)=e(\phi) * e(\psi)$,

- $e(\phi \rightarrow \psi)=e(\phi) \Rightarrow e(\psi)$ and

- $e(\overline{0})=0$.

If an $\mathscr{L}$-evaluation $e$ satisfies $e(\chi)=1$ for every $\chi$ in $\Gamma$, it is called an $\mathscr{L}$-model ${ }^{7}$ for $\Gamma$. We write $\Gamma \models \mathscr{L} \phi$ if $e(\phi)=1$ for all $\mathscr{L}$-models $e$ for $\Gamma$. If $\Gamma$ is empty, we simply write $\models \mathscr{L} \phi$ instead of $\emptyset \models \mathscr{L} \phi$. FL-formulae $\phi$ for which $\models \mathscr{L} \phi$ are called $\mathscr{L}$-tautologies. The relation $\models$ is called semantic consequence.

\footnotetext{
${ }^{6}$ Note that an $\mathscr{L}$-evaluation is completely determined by its action on the propositional variables.

${ }^{7}$ Note that $\mathscr{L}$-models for the empty set are just $\mathscr{L}$-evaluations.
} 
Evaluations form a connection between the connectives of the logic and the algebraic operators in residuated lattices. Note that $e(\overline{1})=e(\overline{0} \rightarrow \overline{0})=e(\overline{0}) \Rightarrow e(\overline{0})=$ $0 \Rightarrow 0=1$ and $e(\neg \phi)=e(\phi \rightarrow \overline{0})=e(\phi) \Rightarrow e(\overline{0})=\neg e(\phi)$.

Now let $\mathscr{C}$ be a class of residuated lattices and $L$ a fuzzy logic.

- We say $L$ is sound w.r.t. $\mathscr{C}$ if for all $\Gamma \subseteq \mathscr{F}_{F L}$ and $\phi \in \mathscr{F}_{F L}, \Gamma \vdash_{L} \phi$ implies $\Gamma=\mathscr{L} \phi$ for all $\mathscr{L}$ in $\mathscr{C}$.

- We say $L$ is complete ${ }^{8}$ w.r.t. $\mathscr{C}$ if for all $\phi \in \mathscr{F}_{F L},(=\mathscr{L} \phi$ for all $\mathscr{L}$ in $\mathscr{C})$ implies $\vdash_{L} \phi$.

- We say $L$ is strong complete w.r.t. $\mathscr{C}$ if for all $\Gamma \subseteq \mathscr{F}_{F L}$ and $\phi \in \mathscr{F}_{F L},(\Gamma \models \mathscr{L} \phi$ for all $\mathscr{L}$ in $\mathscr{C}$ ) implies $\Gamma \vdash_{L} \phi$.

We will illustrate these definitions in the following subsections.

\subsubsection{Monoidal logic}

Monoidal logic (ML) was introduced by Höhle in [26]. Its axioms ${ }^{9}$ are:

$$
\begin{array}{ll}
(M L .1) & (\phi \rightarrow \psi) \rightarrow((\psi \rightarrow \chi) \rightarrow(\phi \rightarrow \chi)), \\
(M L .2) & \phi \rightarrow(\phi \vee \psi), \\
(M L .3) & \psi \rightarrow(\phi \vee \psi), \\
(M L .4) & (\phi \rightarrow \chi) \rightarrow((\psi \rightarrow \chi) \rightarrow((\phi \vee \psi) \rightarrow \chi)), \\
(M L .5) & (\phi \wedge \psi) \rightarrow \phi, \\
(M L .6) & (\phi \wedge \psi) \rightarrow \psi, \\
(M L .7) & (\phi \& \psi) \rightarrow \phi, \\
(M L .8) & (\phi \& \psi) \rightarrow(\psi \& \phi), \\
(M L .9) & (\phi \rightarrow \psi) \rightarrow((\phi \rightarrow \chi) \rightarrow(\phi \rightarrow(\psi \wedge \chi))), \\
(M L .10) & (\phi \rightarrow(\psi \rightarrow \chi)) \rightarrow((\phi \& \psi) \rightarrow \chi), \\
(M L .11) & ((\phi \& \psi) \rightarrow \chi) \rightarrow(\phi \rightarrow(\psi \rightarrow \chi)), \\
(M L .12) & 0 \rightarrow \phi .
\end{array}
$$

This means that for all possible choices of FL-formulae for $\phi, \psi$ and $\chi$, the above FL-formulae are provable in ML. For example, $(\overline{0} \rightarrow q) \rightarrow((p \& q) \vee(\overline{0} \rightarrow q))$ is provable in ML, because it is an instance ${ }^{10}$ of the third axiom, with $\psi=(\overline{0} \rightarrow q)$

\footnotetext{
${ }^{8}$ For every logic $L$ appearing in this chapter, completeness w.r.t. a class $\mathscr{C}$ of residuated lattices (or, in the next sections, triangle algebras) implies that, for every finite theory $\Gamma \subseteq \mathscr{F}_{F L}$ and $\phi \in \mathscr{F}_{F L}$, $\Gamma \vdash_{L} \phi$ if $\Gamma \models \mathscr{L} \phi$ for all $\mathscr{L}$ in $\mathscr{C}$. In other words, completeness implies 'strong completeness for finite theories'.

${ }^{9}$ In [26] there are two axioms instead of the last one. In these two axioms the negation $\neg$ appears (as a unary connective), but not the constant $\overline{0}$. In this work we have chosen another way, namely to define the negation based on the constant $\overline{0}$ (instead of the other way around).

Moreover, also $(\phi \&(\psi \& \chi)) \rightarrow((\phi \& \psi) \& \chi)$ was listed as an axiom. But FL-formulae of this form can be proven from the other axioms, so it can be left out.

${ }^{10} \mathrm{An}$ instance of an axiom of ML is any FL-formula obtained by replacing $\phi, \psi$ and $\chi$ with FLformulae. For example, $\left(\left(p_{1} \rightarrow\left(p_{2} \vee \overline{0}\right)\right) \&\left(p_{2} \wedge q\right)\right) \rightarrow\left(p_{1} \rightarrow\left(p_{2} \vee \overline{0}\right)\right)$ is an instance of $(\phi \& \psi) \rightarrow$ $\phi$.
} 
and $\phi=p \& q$.

To show that other FL-formulae are provable in ML, ML has one deduction rule. This deduction rule is called modus ponens (MP) and states that if $\phi$ and $\phi \rightarrow \psi$ are provable then so is $\psi$. Now we can formally define what a proof in ML of an FL-formula $\phi$ from a theory $\Gamma$ is: it is a finite sequence of FL-formulae in which every FL-formula is either an instance of an axiom of ML, an element of $\Gamma$ or the result of an application of the modus ponens to two FL-formulae appearing earlier in the sequence. If such a proof exists, this is denoted as $\Gamma \vdash_{M L} \phi$. If $\Gamma$ is empty, we simply write $\vdash_{M L} \phi$ instead of $\emptyset \vdash_{M L} \phi$. An important result about ML is its soundness and completeness w.r.t. residuated lattices.

Theorem 2. [26] Monoidal logic is sound and strong complete w.r.t. residuated lattices. In other words: for all $\Gamma \subseteq \mathscr{F}_{F L}$ and $\phi \in \mathscr{F}_{F L}$, we have $\Gamma \vdash_{M L} \phi$ iff $\Gamma \models \mathscr{L} \phi$ for all residuated lattices $\mathscr{L}$.

Each identity or inequality that is valid in residuated lattices can easily be transformed into a scheme ${ }^{11}$ of FL-formulae that are provable in ML. Therefore we just need to change such an identity or inequality to an equivalent 'equal to 1 identity'.

- An example with an identity: $x \Rightarrow(y \sqcap z)=(x \Rightarrow y) \sqcap(x \Rightarrow z)$ holds in all residuated lattices. This is equivalent with $(x \Rightarrow(y \sqcap z)) \Leftrightarrow((x \Rightarrow y) \sqcap(x \Rightarrow z))=1$, which can be immediately transformed into the scheme $(\phi \rightarrow(\psi \wedge \chi)) \leftrightarrow((\phi \rightarrow$ $\psi) \wedge(\phi \rightarrow \chi))$ of FL-formulae that are provable in ML.

- An example with an inequality: $x * y \leq x \sqcap y$ holds in all residuated lattices. This is equivalent with $(x * y) \Rightarrow(x \sqcap y)=1$, which can be transformed into the scheme $(\phi \& \psi) \rightarrow(\phi \wedge \psi)$ of FL-formulae that are provable in ML.

So there is a close connection between identities (and inequalities) in residuated lattices and (schemes of) FL-formulae that are provable in ML.

ML enjoys a so-called local deduction theorem:

Theorem 3. [24] Let $\Gamma \cup\{\phi, \psi\}$ be a set of FL-formulae. Then the following are equivalent:

- $\Gamma \cup\{\phi\} \vdash{ }_{M L} \psi$,

- there is an integer $n$ such that $\Gamma \vdash_{M L} \phi^{n} \rightarrow \psi$.

This local deduction theorem, as well as the soundness and completeness of ML, remain valid in axiomatic extensions of ML. An axiomatic extension of ML is a logic having the same axioms and deduction rule as ML, plus one or more other axioms. Axiomatic extensions of ML are sound and complete w.r.t. residuated lattices that satisfy the identities corresponding to the extra axioms.

${ }^{11}$ A scheme of FL-formulae consists of all FL-formulae of a particular form (the instances of the scheme). Note that the axioms of ML are schemes. 


\subsubsection{Monoidal t-norm based logic}

Monoidal t-norm based logic (MTL) [16] is an axiomatic extension of ML. The extra axiom is $(\phi \rightarrow \psi) \vee(\psi \rightarrow \phi)$. This axiom corresponds with the identity $(x \Rightarrow$ $y) \sqcup(y \Rightarrow x)=1$ (prelinearity) in residuated lattices. Therefore we have the following soundness and completeness theorem.

Theorem 4. [16] Monoidal t-norm based logic is sound and strong complete w.r.t. prelinear residuated lattices (MTL-algebras). In other words: for all $\Gamma \subseteq \mathscr{F}_{F L}$ and $\phi \in \mathscr{F}_{F L}$, we have $\Gamma \vdash_{M T L} \phi$ iff $\Gamma \models \mathscr{L} \phi$ for all MTL-algebras $\mathscr{L}$.

The provability relation $\vdash_{M T L}$ is defined in the same way as $\vdash_{M L}$, only now also instances of the axiom $(\phi \rightarrow \psi) \vee(\psi \rightarrow \phi)$ may appear in proofs of FL-formulae. Because MTL-algebras are isomorphic to subdirect products of MTL-chains, the strong completeness can be strengthened to so-called (strong) chain completeness, i.e., (strong) completeness w.r.t. MTL-chains.

Theorem 5. [16] Monoidal t-norm based logic is sound and strong chain complete. In other words: for all $\Gamma \subseteq \mathscr{F}_{F L}$ and $\phi \in \mathscr{F}_{F L}$, we have $\Gamma \vdash_{M T L} \phi$ iff $\Gamma \models_{\mathscr{L}} \phi$ for all MTL-chains $\mathscr{L}$.

Chain completeness and strong chain completeness are properties that remain valid for axiomatic extensions of MTL. Important theorems about MTL that do not necessarily remain valid for axiomatic extensions, are standard completeness and strong standard completeness. Recall that standard MTL-algebras are MTL-algebras on the unit interval.

Theorem 6. [29] Monoidal t-norm based logic is sound and strong standard complete. In other words: for all $\Gamma \subseteq \mathscr{F}_{F L}$ and $\phi \in \mathscr{F}_{F L}$, we have $\Gamma \vdash_{M T L} \phi$ iff $\Gamma \models \mathscr{L} \phi$ for all standard MTL-chains $\mathscr{L}$.

Because of this theorem, and because standard MTL-chains are induced by leftcontinuous t-norms, we can say that MTL is 'the logic of left-continuous t-norms'.

MTL enjoys the same local deduction theorem as ML:

Theorem 7. [24] Let $\Gamma \cup\{\phi, \psi\}$ be a set of FL-formulae. Then the following are equivalent:

- $\Gamma \cup\{\phi\} \vdash_{M T L} \psi$,

- there is an integer $n$ such that $\Gamma \vdash_{M T L} \phi^{n} \rightarrow \psi$.

\subsubsection{Basic logic}

Basic logic (BL) [24] is an axiomatic extension of MTL (and therefore also of ML). The extra axiom is $(\phi \wedge \psi) \rightarrow(\phi \&(\phi \rightarrow \psi))$. This axiom corresponds with the identity $(x \sqcap y) \Rightarrow(x *(x \Rightarrow y))=1$ in residuated lattices, in other words $x \sqcap y \leq$ $x *(x \Rightarrow y)$. This is equivalent with $x \sqcap y=x *(x \Rightarrow y)$ (divisibility) because $x \sqcap y \geq$ $x *(x \Rightarrow y)$ is valid in all residuated lattices. Because BL is an axiomatic extension 
of MTL and because divisible MTL-algebras are BL-algebras, we immediately have the following soundness and completeness result.

Theorem 8. [24] Basic logic is sound and strong chain complete. In other words: for all $\Gamma \subseteq \mathscr{F}_{F L}$ and $\phi \in \mathscr{F}_{F L}$, we have $\Gamma \vdash_{B L} \phi$ iff $\Gamma=\mathscr{L} \phi$ for all BL-chains $\mathscr{L}$.

Note that BL is also (strong) complete w.r.t. all BL-algebras, which is a weaker property than (strong) chain completeness. BL also satisfies standard completeness (which is stronger than chain completeness), but not strong standard completeness. This implies that the following theorem in general does not hold for infinite theories $\Gamma$.

Theorem 9. [5] For all finite $\Gamma \subseteq \mathscr{F}_{F L}$ and $\phi \in \mathscr{F}_{F L}$, we have $\Gamma \vdash_{B L} \phi$ iff $\Gamma \models \mathscr{L} \phi$ for all standard BL-chains $\mathscr{L}$.

Because standard BL-chains are induced by continuous t-norms, we can say BL is 'the logic of continuous t-norms'.

The local deduction theorem is also valid for BL.

Theorem 10. [24] Let $\Gamma \cup\{\phi, \psi\}$ be a set of FL-formulae. Then the following are equivalent:

- $\Gamma \cup\{\phi\} \vdash_{B L} \psi$,

- there is an integer $n$ such that $\Gamma \vdash_{B L} \phi^{n} \rightarrow \psi$.

\subsubsection{Lukasiewicz logic}

Łukasiewicz logic ( $\mathrm{E}$ ) is an axiomatic extension of BL (and therefore also of MTL and ML). The extra axiom is $\neg \neg \phi \rightarrow \phi$, in which $\neg \phi$ is a short notation for $\phi \rightarrow \overline{0}$. This axiom corresponds with the identity $\neg \neg x \Rightarrow x=1$ in residuated lattices, in other words $\neg \neg x \leq x$. This is equivalent with $\neg \neg x=x$ (involutive negation) because $x \leq \neg \neg x$ is valid in all residuated lattices. Because $€$ is an axiomatic extension of MTL, we immediately have the following soundness and completeness result.

Theorem 11. [24] Łukasiewicz logic is sound and strong chain complete. In other words: for all $\Gamma \subseteq \mathscr{F}_{F L}$ and $\phi \in \mathscr{F}_{F L}$, we have $\Gamma \vdash_{t} \phi$ iff $\Gamma \models_{E} \phi$ for all $M V$-chains $\mathscr{L}$.

Note that $€$ is also (strong) complete w.r.t. all MV-algebras, which is a weaker property than (strong) chain completeness. $€$ also satisfies standard completeness (which is stronger than chain completeness), but not strong standard completeness. This implies that the following theorem in general does not hold for infinite theories.

Theorem 12. [3, 24] For all finite $\Gamma \subseteq \mathscr{F}_{F L}$ and $\phi \in \mathscr{F}_{F L}$, we have $\Gamma \vdash_{E} \phi$ iff $\Gamma \models \mathscr{L} \phi$ for all standard $M V$-chains $\mathscr{L}$.

Because standard MV-chains are induced by t-norms that are conjugated to the Łukasiewicz t-norm, we can say that $€$ is 'the logic of the Łukasiewicz t-norm'.

The local deduction theorem is also valid for $€$. 
Theorem 13. [24] Let $\Gamma \cup\{\phi, \psi\}$ be a set of FL-formulae. Then the following are equivalent:

- $\Gamma \cup\{\phi\} \vdash_{\ell} \psi$,

- there is an integer $n$ such that $\Gamma \vdash_{\ell} \phi^{n} \rightarrow \psi$.

\subsubsection{Classical logic}

Classical logic $\left(\mathrm{CPC}^{12}\right)$ is an axiomatic extension of $€$ (and therefore also of BL, MTL and ML). The extra axiom is $\phi \rightarrow(\phi \& \phi)$. This axiom corresponds with the identity $x \Rightarrow(x * x)=1$ in residuated lattices, in other words $x \leq x * x$. This is equivalent with $x=x * x$ (contraction) because $x * x \leq x$ is valid in all residuated lattices. Because CPC is an axiomatic extension of MTL, we immediately have the following soundness and completeness result.

Theorem 14. [34] Classical logic is sound and strong chain complete. In other words: for all $\Gamma \subseteq \mathscr{F}_{F L}$ and $\phi \in \mathscr{F}_{F L}$, we have $\Gamma \vdash_{C P C} \phi$ iff $\Gamma=\mathscr{L} \phi$ for all linear Boolean algebras $\mathscr{L}$.

Note that there is only one linear Boolean algebra (apart from the trivial one with one element), namely the Boolean algebra with two elements, 0 and 1. Therefore CPC cannot satisfy standard completeness, in the sense that CPC is not complete w.r.t. Boolean algebras on the unit interval (because there are no such Boolean algebras).

The local deduction theorem is also valid for CPC. But because in CPC the FLformulae $\phi$ and $\phi^{n}$ (with $n$ a strictly positive integer) are equivalent (meaning $\vdash_{C P C}$ $\phi \leftrightarrow \phi^{n}$ holds $\left.^{13}\right)$, the theorem can be strengthened.

Theorem 15. [34] Let $\Gamma \cup\{\phi, \psi\}$ be a set of FL-formulae. Then the following are equivalent:

- $\Gamma \cup\{\phi\} \vdash_{C P C} \psi$,

- $\Gamma \vdash_{C P C} \phi \rightarrow \psi$.

\subsubsection{Other fuzzy logics}

Apart from the examples in the previous sections, many other fuzzy logics can be defined by adding axioms to ML, MTL,... Examples can be found in e.g. [6]). In [6] it was proven that an axiomatic extension of MTL is strong standard complete iff it has the real-chain embedding property, i.e., iff each countable MTL-chain in its semantics is embeddable in a standard MTL-chain in its semantics.

\footnotetext{
12 The abbreviation, taken from [6], stands for 'classical propositional calculus'.

${ }^{13}$ In the logics we are concerned with in this work, we have the following property. If a subformula of a formula is replaced by an equivalent subformula, the resulting formula is equivalent with the original one. This can be proven using soundness and completeness. For example, in ML and its axiomatic extensions $\left(p_{1} \& p_{2}\right) \rightarrow q$ is equivalent with $\left(p_{2} \& p_{1}\right) \rightarrow q$ because the subformulae $p_{1} \& p_{2}$ and $p_{2} \& p_{1}$ are equivalent.
} 


\subsection{Interval-Valued Monoidal Logic: Definition}

As semantics of interval-valued fuzzy logics, we choose triangle algebras. Because triangle algebras have more operators than residuated lattices, IVFL-formulae can contain more connectives than FL-formulae.

Definition 10. [41] IVFL-formulae are built up from a countable set of propositional variables (denoted by $p, q, r, p_{1}, p_{2}, \ldots$ ) and the constants $\overline{0}$ and $\bar{u}$. These symbols are IVFL-formulae by definition. The other IVFL-formulae are defined recursively: if $\phi$ and $\psi$ are IVFL-formulae, then so are $(\phi \wedge \psi),(\phi \vee \psi),(\phi \& \psi),(\phi \rightarrow \psi), \square \phi$ and $\diamond \phi$. The set of IVFL-formulae is denoted by $\mathscr{F}_{I V F L}$. Note that $\mathscr{F}_{F L} \subseteq \mathscr{F}_{I V F L}$.

In order to avoid unnecessary brackets, we agree on the following priority rules:

- unary operators always take precedence over binary ones, while

- among the connectives, \& has the highest priority; furthermore $\wedge$ and $\vee$ take precedence over $\rightarrow$,

- the outermost brackets are not written.

The same notations $(\overline{1}$ is $\overline{0} \rightarrow \overline{0}, \ldots)$ as for FL-formulae are used. Now we are ready to introduce interval-valued monoidal logic (IVML) [41]. Its axioms are those of ML, i.e., (ML.1)-(ML.12), complemented with axioms corresponding to 13 properties of triangle algebras:

$$
\begin{aligned}
& (\text { IVML.2) } \square \phi \rightarrow \square \square \phi, \\
& (I V M L .3) \quad(\square \phi \wedge \square \psi) \rightarrow \square(\phi \wedge \psi), \quad\left(I V M L .3^{\prime}\right)(\diamond \phi \wedge \diamond \psi) \rightarrow \diamond(\phi \wedge \psi), \\
& (I V M L .4) \quad \square(\phi \vee \psi) \rightarrow(\square \phi \vee \square \psi), \quad\left(I V M L .4^{\prime}\right) \diamond(\phi \vee \psi) \rightarrow(\diamond \phi \vee \diamond \psi), \\
& (I V M L .5) \quad \neg \square \bar{u}, \\
& (I V M L .6) \quad \diamond \phi \rightarrow \square \diamond \phi, \\
& (I V M L .7) \quad \square(\phi \rightarrow \psi) \rightarrow(\square \phi \rightarrow \square \psi), \\
& (I V M L .9) \quad(\square \phi \rightarrow \square \psi) \rightarrow \square(\square \phi \rightarrow \square \psi), \\
& \text { (IVML.10) } \phi \leftrightarrow(\square \phi \vee(\diamond \phi \wedge \bar{u})) \\
& \left(I V M L .1^{\prime}\right) \phi \rightarrow \diamond \phi \\
& \begin{array}{l}
\left(I V M L .4^{\prime}\right) \diamond(\phi \vee \\
\left(I V M L .5^{\prime}\right) \diamond \bar{u},
\end{array} \\
& \left(I V M L .6^{\prime}\right) \diamond \square \phi \rightarrow \square \phi \text {, }
\end{aligned}
$$

All instances of these axioms are by definition provable in IVML. To determine which other IVFL-formulae are provable, there are three deduction rules: modus ponens (MP, if $\phi$ and $\phi \rightarrow \psi$ are provable in IVML, then so is $\psi$ ), generalization ( G, if $\phi$ is provable in IVML, then so is $\square \phi$ ) and monotonicity of $\diamond(\mathrm{M} \diamond$, if $\phi \rightarrow \psi$ is provable, then so is $\nabla \phi \rightarrow \diamond \psi)$. Proofs in IVML and the provability relation $\vdash_{I V M L}$ are defined in the usual way, similarly as for ML (and the other fuzzy logics from Section 4.1). If $\Gamma$ is a theory, i.e., a set of IVFL-formulae, then a (formal) proof of an IVFL-formula $\phi$ in $\Gamma$ is a finite sequence of IVFL-formulae with $\phi$ at its end, such that every IVFL-formula in the sequence is either an instance of an axiom of IVML, an IVFL-formula of $\Gamma$, or the result of an application of a deduction rule to previous IVFL-formulae in the sequence. If a proof for $\phi$ exists in $\Gamma$, we denote this by $\Gamma \vdash_{I V M L} \phi$. 
Definition 11. [41] Let $\mathscr{A}=(A, \sqcap, \sqcup, *, \Rightarrow, v, \mu, 0, u, 1)$ be a triangle algebra, $\Gamma$ a theory (i.e., a set of IVFL-formulae). An $\mathscr{A}$-evaluation is a mapping $e$ from the set of IVFL-formulae to $A$ that satisfies, for each two IVFL-formulae $\phi$ and $\psi$ :

- $e(\phi \wedge \psi)=e(\phi) \sqcap e(\psi)$,

- $e(\phi \vee \psi)=e(\phi) \sqcup e(\psi)$,

- $e(\phi \& \psi)=e(\phi) * e(\psi)$,

- $e(\phi \rightarrow \psi)=e(\phi) \Rightarrow e(\psi)$,

- $e(\square \phi)=v e(\phi)$,

- $e(\diamond \phi)=\mu e(\phi)$,

- $e(\overline{0})=0$ and

- $e(\bar{u})=u$.

If an $\mathscr{A}$-evaluation $e$ satisfies $e(\chi)=1$ for every $\chi$ in $\Gamma$, it is called an $\mathscr{A}$-model for $\Gamma$. We write $\Gamma=_{\mathscr{A}} \phi$ if $e(\phi)=1$ for all $\mathscr{A}$-models $e$ for $\Gamma$.

Soundness, completeness and strong completeness are defined similarly as for formal fuzzy logics. We just have to replace 'residuated lattice' by 'triangle algebra' and 'FL-formula' by 'IVFL-formula'.

Now we introduce some axiomatic extensions of IVML, by adding well-known axioms. Note that these axioms are applied to IVFL-formulae of the form $\square \phi$ instead of to all IVFL-formulae. As the image of a triangle algebra $(A, \sqcap, \sqcup, *$, $\Rightarrow, v, \mu, 0, u, 1)$ under $v$ is the set $E(\mathscr{A})$ of exact elements ${ }^{14}$, this means that the axioms schemes do not hold for all truth values, but only for exact truth values. This is not a drawback. On the contrary, it is precisely what we want because the exact truth values are easier to interpret and handle. Moreover, using Proposition 3, for all axioms equivalent axioms can be found that only involve IVFL-formulae of the form $\square \phi$ and $\diamond \phi$, and $\bar{u}$.

Definition 12. [38]

- Interval-valued monoidal t-norm based logic (IVMTL) is IVML extended with the axiom scheme pseudo-prelinearity

$$
(\square \phi \rightarrow \square \psi) \vee(\square \psi \rightarrow \square \phi) .
$$

- Interval-valued basic logic (IVBL) is IVMTL extended with the axiom scheme pseudo-divisibility

$$
(\square \phi \wedge \square \psi) \rightarrow(\square \phi \&(\square \phi \rightarrow \square \psi)) .
$$

- Interval-valued Łukasiewicz logic (IVŁ) is IVBL extended with the axiom scheme pseudo-involution

$$
\neg \neg \square \phi \rightarrow \square \phi .
$$

\footnotetext{
${ }^{14}$ Note that the image under $\mu$ is also $E(\mathscr{A})$. All axiom schemes in Definition 12 can also be given in an equivalent way by changing $\square \phi$ to $\diamond \phi$ and/or $\square \psi$ to $\diamond \psi$.
} 
- Interval-valued classical propositional calculus (IVCPC) is IVŁ extended with the axiom scheme pseudo-contraction

$$
\square \phi \rightarrow(\square \phi \& \square \phi) .
$$

\subsection{Soundness and completeness}

It is easy to check that IVML is sound w.r.t. the variety of triangle algebras, i.e., that if an IVFL-formula $\phi$ can be proven from a theory $\Gamma$ in IVML $\left(\Gamma \vdash_{I V M L} \phi\right)$, then for every triangle algebra $\mathscr{A}$ and for every $\mathscr{A}$-model $e$ of $\Gamma, e(\phi)=1$ (in other words: for every triangle algebra $\mathscr{A}, \Gamma=_{\mathscr{A}} \phi$ ). To show that IVML is also strong complete (w.r.t. triangle algebras), i.e., that the converse of soundness also holds, a general result from abstract algebraic logic (shortly AAL, see e.g. [19] for a survey) can be applied. It proceeds by showing that IVML is an implicative logic (in the sense of Rasiowa [33]). From this we can deduce (according to e.g. [18]) that IVML is strong complete w.r.t. the variety of triangle algebras if it is sound w.r.t. it and if in triangle algebras $x=y$ if $x \Rightarrow y=1$ and $y \Rightarrow x=1$. Triangle algebras indeed satisfy these conditions, so we can conclude that IVML is sound and strong complete w.r.t. triangle algebras.

Theorem 16. (Soundness and strong completeness of IVML) [41] An IVFLformula $\phi$ can be deduced from a theory $\Gamma$ in IVML iff for every triangle algebra $\mathscr{A}$ and for every $\mathscr{A}$-model $e$ of $\Gamma, e(\phi)=1$.

A more basic proof for the completeness of IVML, using the Lindenbaum algebra of IVML and inspired by a similar, commonly used procedure for formal fuzzy logics, was given in [36].

Theorem 16 implies similar results for axiomatic extensions (e.g. the intervalvalued fuzzy logics in Definition 12), in the same way as the soundness and completeness of ML remains valid for axiomatic extensions. This can be seen by taking the set of all instances of the extra axioms as $\Gamma$ in Theorem 16. In particular, all extensions of IVML introduced in Section 4.2 are sound and (strong) complete w.r.t. their corresponding subvariety of the variety of triangle algebras. For example, IVBL is sound and complete w.r.t. the variety of triangle algebras satisfying $(v x \Rightarrow v y) \sqcup(v y \Rightarrow v x)=1$ and $v x \sqcap v y \leq v x *(v x \Rightarrow v y)$.

For IVMTL and its axiomatic extensions we can prove a stronger version of completeness, namely strong pseudo-chain completeness. This is similar to axiomatic extensions of MTL being strong chain-complete. Together with Theorem 16, Theorem 1 implies the following result:

Theorem 17. [42] For each set of IVFL-formulae $\Gamma \cup \phi$, the following three statements are equivalent:

- $\phi$ can be deduced from a theory $\Gamma$ in $\operatorname{IVMTL}\left(\Gamma \vdash_{I V M T L} \phi\right)$, 
- for every pseudo-prelinear triangle algebra $\mathscr{A}$ and for every $\mathscr{A}$-model e of $\Gamma$, $e(\phi)=1$,

- for every pseudo-linear triangle algebra $\mathscr{A}$ and for every $\mathscr{A}$-model e of $\Gamma$, $e(\phi)=1$.

This completeness result remains valid for axiomatic extensions of IVMTL. The reason is that Theorem 1 also holds for subvarieties of pseudo-prelinear triangle algebras.

Using Theorem 6 and the real-chain embedding property, also the strong standard completeness of IVMTL can be proven.

Theorem 18. [38] (Strong standard completeness) For each set of IVFL-formulae $\Gamma \cup\{\phi\}$, the following four statements are equivalent:

1. $\phi$ can be deduced from $\Gamma$ in IVMTL $\left(\Gamma \vdash_{I V M T L} \phi\right)$,

2. for every pseudo-prelinear triangle algebra $\mathscr{A}, \Gamma=\mathscr{A} \phi$ (i.e., for every $\mathscr{A}$-model e of $\Gamma, e(\phi)=1)$,

3. for every pseudo-linear triangle algebra $\mathscr{A}, \Gamma \models_{\mathscr{A}} \phi$,

4. for every standard triangle algebra $\mathscr{A}, \Gamma=\mathscr{A} \phi$.

Because of Proposition 2, every standard triangle algebra is isomorphic to a standard extended IVRL, and every standard extended IVRL is a standard triangle algebra. This result leads to the following corollary of Theorem 18 .

Corollary 1. For each set of IVFL-formulae $\Gamma \cup\{\phi\}$, the following statements are equivalent:

1. $\phi$ can be deduced from $\Gamma$ in IVMTL $\left(\Gamma \vdash_{I V M T L} \phi\right)$,

2. for every standard extended IVRL $\mathscr{A}, \Gamma=\mathscr{A} \phi$.

So we can truly state that IVMTL is an interval-valued fuzzy logic. It is the logic of the t-norms $\mathscr{T}_{T, t}$ in (2), with $T$ a left-continuous t-norm on the unit interval.

Finally, it is also possible to prove the local deduction theorem for IVML (and its axiomatic extensions), which gives a connection between $\vdash_{\mathbf{L}}$ and $\rightarrow$.

Theorem 19. [38] Let $\Gamma \cup\{\phi, \psi\}$ be a set of IVFL-formulae, and $\boldsymbol{L}$ be an axiomatic extension of IVML. Then the following are equivalent:

- $\Gamma \cup\{\phi\} \vdash_{L} \psi$,

- There is an integer $n$ such that $\Gamma \vdash_{\boldsymbol{L}}(\square \phi)^{n} \rightarrow \psi$.

\subsection{Interpretation}

Interval-valued fuzzy logics as we introduced them, are truth-functional logics: the truth degree of a compound proposition is determined by the truth degree of its constituent parts. This causes some counterintuitive results, if we want to interpret the element $[0,1]$ of an IVRL as uncertainty regarding the actual truth value of a 
proposition. For example: suppose we don't know anything about the truth value of propositions $p$ and $q$, i.e., $e(p)=e(q)=[0,1]$. Then yet the implication $p \rightarrow q$ is definitely valid: $e(p \rightarrow q)=e(p) \Rightarrow e(q)=[1,1]$. However, if $\neg[0,1]=[0,1] 15$ (which is intuitively preferable, since the negation of an uncertain proposition is still uncertain), then we can take $q=\neg p$, and obtain that $p \rightarrow \neg p$ is true. Or, equivalently (using the residuation principle), that $p \& p$ is false. This does not seem intuitive, as one would rather expect $p \& p$ to be uncertain if $p$ is uncertain.

Another consequence of $[0,1] \Rightarrow[0,1]=[1,1]$ is that it is impossible to interpret the intervals as a set in which the 'real' (unknown) truth value is contained, and $X \Rightarrow Y$ as the smallest closed interval containing every $x \Rightarrow y$, with $x$ in $X$ and $y$ in $Y$ (as in [15]). Indeed: $1 \in[0,1]$ and $0 \in[0,1]$, but $1 \Rightarrow 0=0 \notin[1,1]$.

On the other hand, for t-norms it is possible that $X * Y$ is the smallest closed interval containing every $x * y$, with $x$ in $X$ and $y$ in $Y$, but only if they are t-representable (described by the axiom $\mu(x * y)=\mu x * \mu y$ ). However, in this case $\neg[0,1]=[0,0]$, which does not seem intuitive ('the negation of an uncertain proposition is absolutely false').

These considerations seem to suggest that IVML and its axiomatic extensions are not suitable to reason with uncertainty. This does not mean that intervals are not a good way for representing degrees of uncertainty, only that they are not suitable as truth values in a truth-functional logical calculus when we interpret them as expressing uncertainty. It might even be impossible to model uncertainty as a truth value in any truth-functional logic. This question is discussed in [12, 13]. However, nothing prevents the intervals in interval-valued fuzzy logics from having more adequate interpretations.

Acknowledgements Bart Van Gasse and Chris Cornelis would like to thank the Research Foundation-Flanders for funding their research.

\section{References}

1. Alcalde, C., Burusco, A., Fuentes-González, R.: A constructive method for the definition of interval-valued fuzzy implication operators. Fuzzy Sets and Systems 153, 211-227 (2005)

2. Bustince, H.: Indicator of inclusion grade for interval-valued fuzzy sets. application to approximate reasoning based on interval-valued fuzzy sets. International Journal of Approximate Reasoning 23, 137-209 (2000)

3. Chang, C.: Algebraic analysis of many valued logics. Transactions of the American Mathematical Society 88(2), 467-490 (1958)

4. Chang, C.: A new proof of the completeness of the lukasiewicz axioms. Transactions of the American Mathematical Society 93(1), 74-80 (1959)

5. Cignoli, R., Esteva, F., Godo, L., Torrens, A.: Basic fuzzy logic is the logic of continuous t-norms and their residua. Soft Computing 4, 106-112 (2000)

6. Cintula, P., Esteva, F., Gispert, J., Godo, L., Montagna, F., Noguera, C.: Distinguished algebraic semantics for t-norm based fuzzy logics: methods and algebraic equivalencies. Annals of Pure and Applied Logic 160(1), 53-81 (2009)

\footnotetext{
15 This is for example the case if $\neg$ is involutive.
} 
7. Cornelis, C., Deschrijver, G., Kerre, E.: Implication in intuitionistic fuzzy and interval-valued fuzzy set theory: construction, classification, application. International Journal of Approximate Reasoning 35, 55-95 (2004)

8. Cornelis, C., Deschrijver, G., Kerre, E.: Advances and challenges in interval-valued fuzzy logic. Fuzzy Sets and Systems 157(5), 622-627 (2006)

9. Deschrijver, G.: The łukasiewicz t-norm in interval-valued fuzzy and intuitionistic fuzzy set theory. In: K.T. Atanassov, J. Kacprzyk, M. Krawczak, E. Szmidt (eds.) Issues in the Representation and Processing of Uncertain and Imprecise Information. Fuzzy Sets, Intuitionistic Fuzzy Sets, Generalized Nets, and Related Topics, pp. 83-101. Akademicka Oficyna Wydawnicza EXIT (2005)

10. Deschrijver, G., Cornelis, C., Kerre, E.: On the representation of intuitionistic fuzzy t-norms and t-conorms. IEEE Transactions on Fuzzy Systems 12, 45-61 (2004)

11. Deschrijver, G., Kerre, E.: Classes of intuitionistic fuzzy t-norms satisfying the residuation principle. International Journal of Uncertainty, Fuzziness and Knowledge-Based Systems 11, 691-709 (2003)

12. Dubois, D.: On ignorance and contradiction considered as truth values (2006). Personal communication

13. Dubois, D., Prade, H.: Can we enforce full compositionality in uncertainty calculi? In: Proceedings of the 11th National Conference on Artificial Intelligence (AAAI'94), pp. 149-154. Seattle, Washington (1994)

14. Dummett, M.: A propositional calculus with denumerable matrix. The Journal of Symbolic Logic 24(2), 97-106 (1959)

15. Esteva, F., Garcia-Calvés, P., Godo, L.: Enriched interval bilattices and partial many-valued logics: an approach to deal with graded truth and imprecision. International Journal of Uncertainty, Fuzziness and Knowledge-Based Systems 2(1), 37-54 (1994)

16. Esteva, F., Godo, L.: Monoidal t-norm based logic: towards a logic for left-continuous t-norms. Fuzzy Sets and Systems 124, 271-288 (2001)

17. Esteva, F., Godo, L., Garcia-Cerdaña, A.: On the hierarchy of t-norm based residuated fuzzy logics. In: M. Fitting, E. Orlowska (eds.) Beyond Two: Theory and Applications of Multiple Valued Logic, pp. 251-272. Physica-Verlag (2003)

18. Font, J.: Beyond rasiowa's algebraic approach to non-classical logics. Studia Logica 82(2), 172-209 (2006)

19. Font, J., Jansana, R., Pigozzi, D.: A survey of abstract algebraic logic. Studia Logica 74, 13-79 (2003)

20. Font, J., Rodriguez, A., Torrens, A.: Wajsberg algebras. Stochastica 8, 5-31 (1984)

21. Gehrke, M., Walker, C., Walker, E.: Some comments on interval-valued fuzzy sets. International Journal of Intelligent Systems 11, 751-759 (1996)

22. Gödel, K.: Zum intuitionistischen aussagenkalkül. Anzeiger der Akademie der Wissenschaften in Wien pp. 65-66 (1932)

23. Gottwald, S.: Mathematical fuzzy logic as a tool for the treatment of vague information. Information Sciences 172, 41-71 (2005)

24. Hájek, P.: Metamathematics of Fuzzy Logic. Trends in Logic—Studia Logica Library. Kluwer Academic Publishers (1998)

25. Heyting, A.: Die formalen Regeln der intuitionistischen Logik. Sitzungsberichte der preuszischen Akademie der Wissenschaften, physikalisch-mathematische Klasse pp. 42-56, 57-71, $158-169(1930)$

26. Höhle, U.: Commutative, residuated l-monoids. In: U. Höhle, E. Klement (eds.) Non-classical Logics and their Applications to Fuzzy Subsets: a Handbook of the Mathematical Foundations of Fuzzy Set Theory, pp. 53-106. Kluwer Academic Publishers (1995)

27. Huntington, E.: Sets of independent postulates for the algebra of logic. Transactions of the American Mathematical Society 5, 288-309 (1904)

28. Jenei, S.: A more efficient method for defining fuzzy connectives. Fuzzy Sets and Systems 90, 25-35 (1997)

29. Jenei, S., Montagna, F.: A proof of standard completeness for Esteva and Godo's logic MTL. Studia Logica 70, 1-10 (2002) 
30. Łukasiewicz, J., Tarski, A.: Untersuchungen über den Aussagenkalkül. Comptes Rendus de la Société des Sciences et des Lettres de Varsovie pp. 1-21 (1930)

31. Ohnishi, M., Matsumoto, K.: Gentzen method in modal calculi, part I. Osaka Mathematical Journal 9, 113-130 (1957)

32. Ohnishi, M., Matsumoto, K.: Gentzen method in modal calculi, part II. Osaka Mathematical Journal 11, 115-120 (1959)

33. Rasiowa, H.: An algebraic approach to non-classical logics. Studies in Logic and the Foundations of Mathematics 78 (1974)

34. Shoenfield, J.: Mathematical logic (1967)

35. Turunen, E.: Mathematics behind Fuzzy Logic. Physica-Verlag (1999)

36. Van Gasse, B.: Interval-valued algebras and fuzzy logics. Ph. D. Thesis, Ghent University, Belgium (2010)

37. Van Gasse, B., Cornelis, C., Deschrijver, G., Kerre, E.: Filters of residuated lattices and triangle algebras. Information Sciences Accepted

38. Van Gasse, B., Cornelis, C., Deschrijver, G., Kerre, E.: The standard completeness of intervalvalued monoidal t-norm based logic. Information Sciences Under review

39. Van Gasse, B., Cornelis, C., Deschrijver, G., Kerre, E.: Triangle algebras: towards an axiomatization of interval-valued residuated lattices. In: Lecture Notes in Computer Science, Proceedings of the 5th International Conference on Rough Sets and Current Trends in Computing (RSCTC 2006), pp. 117-126 (2006)

40. Van Gasse, B., Cornelis, C., Deschrijver, G., Kerre, E.: A characterization of interval-valued residuated lattices. International Journal of Approximate Reasoning 49, 478-487 (2008)

41. Van Gasse, B., Cornelis, C., Deschrijver, G., Kerre, E.: Triangle algebras: A formal logic approach to interval-valued residuated lattices. Fuzzy Sets and Systems 159, 1042-1060 (2008)

42. Van Gasse, B., Cornelis, C., Deschrijver, G., Kerre, E.: The pseudo-linear semantics of interval-valued fuzzy logics. Information Sciences 179, 717-728 (2009)

43. Zadeh, L.: The concept of a linguistic variable and its application to approximate reasoning i. Information Sciences 8, 199-249 (1975)

44. Zalta, E.: Basic concepts in modal logic (1995). http://mally.stanford.edu/notes.pdf 